\title{
Early saccade planning cannot override oculomotor interference elicited by gaze and arrow distractors
}

\author{
Mario Dalmaso $^{1}$ (1) $\cdot$ Luigi Castelli $^{1} \cdot$ Giovanni Galfano $^{1}$
}

Published online: 30 June 2020

(C) The Psychonomic Society, Inc. 2020

\begin{abstract}
Humans tend to perform reflexive saccades according to the eye-gaze direction of other individuals. Here, in two experiments, we tested whether preparing a saccade before the onset of a task-irrelevant averted-gaze stimulus can abolish this form of gazefollowing behavior. At the beginning of each trial, participants received the instruction to prepare for a leftward or a rightward saccade. This was provided either on a trial-by-trial basis (Experiment 1) or was maintained constant within a whole block of trials (Experiment 2). Then, a central fixation spot changed in color, acting as a "go" signal to perform the saccade. Simultaneously with the go-signal onset, a task-irrelevant distractor face looked either leftwards or rightwards. In so doing, no temporal overlapping was likely to occur between saccade preparation and the presentation of the distractor. Arrows were also employed as non-social control stimuli. In both experiments - and regardless of the distractor type - saccadic latencies were smaller when both the instruction and the distractor conveyed the same spatial vector, rather than the opposite. Taken together, these results suggest that an early preparation of saccades is not sufficient to override the oculomotor interference effects elicited by both social and non-social distractors.
\end{abstract}

Keywords Gaze distractor $\cdot$ Arrow distractor $\cdot$ Saccades $\cdot$ Eye movements $\cdot$ Social attention

\section{Introduction}

The notion that our oculomotor system can be profoundly shaped by the presence of eye-gaze stimuli has been known since the pioneering works of Yarbus (1967). In his most classic studies, Yarbus reported the presence of an intense fixational activity focused on the eye region of the individuals depicted in different social scenes (see also, e.g., Tatler, Wade, Kwan, Findlay, \& Velichkovsky, 2010).

Another stream of studies has also reported that avertedgaze faces can elicit, in an observer, an eye movement towards the gazed-at spatial location. Looking where another individual is looking represents an essential ability to establish productive and meaningful relationships with both our conspecific and the environment around us (e.g., Capozzi \& Ristic, 2018; Dalmaso, Castelli, \& Galfano, 2020b). In the lab, this

Mario Dalmaso

mario.dalmaso@unipd.it; mario.dalmaso@gmail.com

1 Department of Developmental and Social Psychology, University of Padova, Via Venezia 8, 35131 Padova, Italy form of overt gaze-following behavior has been mainly documented by adopting the instructed-saccade task developed by Ricciardelli, Bricolo, Aglioti, and Chelazzi (2002). In this task, participants are typically asked to perform a leftward or a rightward saccade according to an instruction cue (e.g., a change in color of the fixation spot, such as blue vs. orange for eliciting a leftward vs. a rightward saccade, respectively) while ignoring a task-irrelevant central face with averted gaze acting as distractor. As a main result, smaller latencies and a greater accuracy typically emerge on trials in which the instruction cue and the distracting face share the same spatial vectors (e.g., right-right; i.e., a congruent condition) rather than the opposite (e.g., right-left; an incongruent condition), thus indicating that averted eye-gaze stimuli can interfere with oculomotor planning and execution pervasively. Outcomes similar to those reported by Ricciardelli et al. (2002) have been documented in subsequent studies employing different types of facial stimuli (e.g., Ciardo, Marino, Actis-Grosso, Rossetti, \& Ricciardelli, 2014; Dalmaso, Alessi, Castelli, \& Galfano, 2020a; Dalmaso, Galfano, \& Castelli, 2015; Porciello, Liuzza, Minio-Paluello, Caprara, \& Aglioti, 2016) but also when directional arrows were employed instead of averted-gaze faces (e.g., Kuhn \& Benson, 2007; Kuhn \& 
Kingstone, 2009), in line with a rich stream of studies that reported similar attentional effects for both gaze and arrow stimuli in healthy individuals (e.g., Galfano et al., 2012; Hermens \& Walker, 2010; Kuhn \& Benson, 2007; Kuhn \& Kingstone, 2009; Tipples, 2008).

Importantly, the interference effect elicited through the task developed by Ricciardelli et al. (2002) appears to be a robust and automatic phenomenon. Indeed, it can be observed when participants are explicitly told that gaze distractors are noninformative with respect to the direction of the instructed saccadic eye movement (e.g., Ricciardelli et al., 2002). In this case, the direction of the gaze stimulus and the direction of the instructed saccade match only on $50 \%$ of trials, and therefore there is no particular incentive in preparing a saccade in the same direction signaled by gaze. However, it is worth noting that all of the studies employing the instructed saccade task (e.g., Kuhn \& Kingstone, 2009; Porciello et al., 2016) provided the instruction cue in close temporal proximity with the onset of the distractor. Furthermore, on each trial, participants had to retrieve from memory the correct association between the instruction cue signal and the direction of the requested saccade. Hence, it is reasonable to assume that the whole chain of processes needed to program and execute a saccade required some time to be fully accomplished. Following this reasoning, one can hypothesize that the interference resulted from the fact that the participants had not completed saccade programming based on the instruction cue when the task-irrelevant distractor was presented. Therefore, the two sources of information (task-relevant and task-irrelevant) were likely to compete in the computation of the response output. In sum, the interference effect emerging from the oculomotor task proposed by Ricciardelli et al. (2002) could be strongly affected by the fact that the distractor is presented within the temporal window in which the saccade is assumed to be prepared. In other words, studies using this paradigm demonstrate that task-irrelevant information - provided by either gaze or arrows - can permeate the key phase of saccadic programming.

The aim of this work was to assess whether the interference effect stemming from the widely used instructed-saccade task can be abolished when the request to program the saccade is provided well in advance with respect to the onset of the distracting stimulus. To this end, we developed a modified version of the task in which the instruction cue was replaced by two distinct stimuli: A "prepare" signal (i.e., an instruction) conveying the spatial vector for programming the saccadic eye movement, and a " $g o$ " signal indicating the need to start executing the saccade. Crucially, the two signals were temporally dissociated, in that they occurred in two separate time windows. More specifically, the signal to prepare for either a rightward or a leftward saccade was given, in isolation, at the beginning of each trial in the form of a direction word (i.e., right vs. left; see also Galfano et al., 2012;
Hommel, Pratt, Colzato, \& Godijn, 2001). Only afterwards, the distracting stimulus appeared alongside the go signal (i.e., a change in color of the fixation spot) to launch the requested saccade. By this means, there was a clear separation between the two crucial time windows in which (1) the instructed saccade was likely to be prepared and (2) the instructed saccade was executed in the presence of the distractor. It should be noted that the procedure adopted in the present study also had the advantage of not requiring the learning of an arbitrary association between symbolic cues and spatial vectors, which can involve considerable effort (Guzzon, Brignani, Miniussi, $\&$ Marzi, 2010). In so doing, the lack of interference exerted by the distractors would lend support to the view that distractors can no longer affect saccade execution once saccadic eye movements have been already programmed. Alternatively, a significant interference effect would be consistent with the view that distractors can hardly be ignored, thus supporting a strong view about their pervasive and likely automatic effects. This task was employed in a first experiment in which the direction word was provided on a trial-bytrial basis. Moreover, two different distractors were used, namely eye gaze versus arrow, to assess potential similarities or differences (also see, e.g., Kuhn \& Benson, 2007; Kuhn \& Kingstone, 2009; Ricciardelli et al., 2002) between the effects triggered by these two types of stimuli.

\section{Experiment 1: Intermixed execution of leftward and rightward saccades}

\section{Methods}

\section{Participants}

The mean sample size used by Ricciardelli et al. (2002) was N = 12 (for similar sample sizes see also Kuhn \& Benson, 2007; Kuhn \& Kingstone, 2009). We stopped at $\mathrm{N}=20$ at the end of a booking session. All participants were students who were naïve to the study (mean age $=24$ years, $S D=1.31$, eight males), had normal or corrected-to-normal vision, and provided written, informed consent. No compensation or credits were provided. The study was approved by the Ethics Committee for Psychological Research at the University of Padova and conducted in accordance with the guidelines laid down in the Declaration of Helsinki.

\section{Apparatus and stimuli}

An EyeLink 1000 Plus (SR Research Ltd, Ottawa, Canada) recorded eye movements monocularly at 1,000 $\mathrm{Hz}$. Participants sat approximately $70 \mathrm{~cm}$ away from a 24 -in. monitor $(1,280 \times 1,024$ pixels, $120 \mathrm{~Hz})$ and a chinrest was used to prevent head movements. A display PC running Experiment 
Builder (SR Research Ltd, Ottawa, Canada) handled timing and stimulus presentation, and a Data Viewer (SR Research Ltd, Ottawa, Canada) was used to extract eye-movement data and to compute saccade amplitude, latency, and direction.

\section{Stimuli and procedure}

Eye-gaze distractor was provided by a female avatar face $\left(10^{\circ}\right.$ width $\times 13^{\circ}$ height) created through DAZ 3D software (https://www.daz3d.com). Three different versions of this face were created: One looking straight, one looking leftwards, and one looking rightwards. An avatar face was employed in order to present participants with a wellcontrolled stimulus with adequate ecological validity. Two white arrows, pointing left or right and of the same size as the eye region of the avatar $\left(1.5^{\circ}\right.$ width $\times 1.2^{\circ}$ height $)$, were employed as non-social control distractors. The background was set to gray $(\mathrm{R}=180, \mathrm{G}=180, \mathrm{~B}=180)$.

Before the experimental session, each participant completed a calibration and a validation procedure. Then, the experiment started. Eye-gaze and arrow distractors were presented in two distinct blocks and block order was counterbalanced across participants. On each trial, a centrally placed direction word (i.e., "SINISTRA" vs. "DESTRA," meaning "LEFT" and "RIGHT," respectively, in Italian in 20-point Arial font) appeared for $1,000 \mathrm{~ms}$ (see Fig. 1). Then, a central black circle (diameter: $.5^{\circ}$ ) appeared and remained visible for the whole duration of the trial. Participants were instructed to look at this spot and then the experimenter initiated the trial through the host PC. This procedure ensured that participants fixated the center of the screen and were allowed to perform a drift- checking procedure. Then, depending on block condition, either a central face with direct gaze or two arrows without the head appeared for 1,000 ms. These were flanked by two black placeholders (side: $.9^{\circ}$ ) placed $9.7^{\circ}$ leftwards and rightwards from the central black circle. After that, the central black circle turned blue $(\mathrm{R}=0, \mathrm{G}=226, \mathrm{~B}=255)$ for $1,000 \mathrm{~ms}$. This change in color acted as the go signal to perform a fast and directionally accurate (i.e., left or right) saccade towards the left or the right placeholder, according to the previously presented direction word. Simultaneously with the color change, the central faces looked either leftwards or rightwards or, in the case of arrows, these pointed either leftwards or rightwards. In so doing, the saccadic eye movement was performed either towards the same location as that indicated by the central stimulus (i.e., a spatially congruent trial) or towards the opposite location (i.e., a spatially incongruent trial). Finally, a blank screen appeared for 1,000 ms. The participants were informed that gaze and arrow stimuli were uninformative with respect to the direction of the instructed saccade. Overall, there were 200 experimental trials. More specifically, in both the gaze and the arrow blocks, there were eight practice and 100 experimental randomized trials, equally distributed in spatially congruent ( 50 trials, 25 with leftward eye movements and 25 with rightward eye movements) and incongruent trials (50 trials, 25 with leftward eye movements and 25 with rightward eye movements).

\section{Data handling and analyses}

Eye movements with a velocity and acceleration exceeding $30 \%$ and $8,000 \% \mathrm{~s}^{2}$, respectively, and with an amplitude

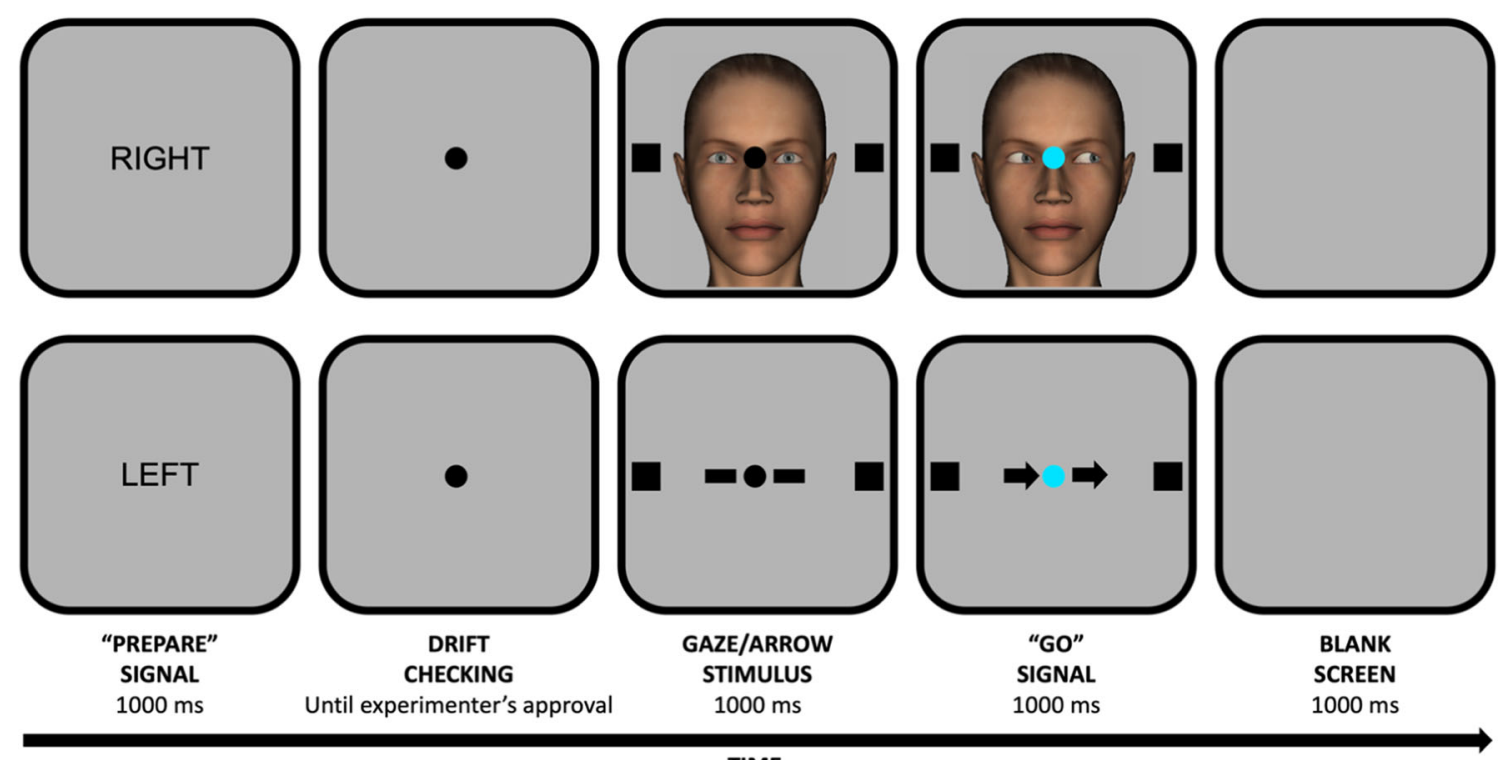

Fig. 1 Example of trials and stimuli (not drawn to scale) employed in both experiments. Upper panel: Example of a spatially congruent trial in which a rightward saccade is required in the presence of a gaze distractor looking rightwards. Lower panel: Example of a spatially incongruent trial in which a leftward saccade is required in the presence of an arrow distractor pointing rightwards 
greater than $2^{\circ}$ were classified as saccades. On each trial, we extracted the first blink-free saccade executed after the onset of the go signal. Latencies of correct saccades - defined as the time elapsed between the go-signal onset and the saccade onset - were markedly positively skewed, and they were therefore $\log$ transformed, in order to obtain more normally distributed data (Howell, 2013). Nevertheless, for the sake of clarity, descriptive statistics are reported as untransformed data. All statistical analyses were performed using JASP (JASP Team, 2020).

Mean saccadic amplitude was $9.39^{\circ}$. Saccades executed towards the opposite location as that conveyed by the direction word (i.e., saccadic errors) were discarded (2.7\% of trials) and analyzed separately. Latencies of correct saccades, and the mean percentage of errors, were analyzed through repeated-measures ANOVAs with Congruency (2: congruent vs. incongruent) and Distractor type (2: gaze vs. arrow) as within-participant factors. Bayesian analyses were also performed for both latencies of correct saccades and errors, in order to estimate which model was more likely supported by the available data. More specifically, Bayesian ANOVAs with Congruency (2: congruent vs. incongruent) and Distractor type (2: gaze vs. arrow) as within-participant factors were carried out.

\section{Results}

With regard to latencies, the main effect of Congruency was significant, $F(1,19)=25.284, p<.001, \eta_{p}^{2}=.571$, with smaller latencies on congruent $(M=235 \mathrm{~ms}, S E=14.99)$ than on incongruent $(M=259 \mathrm{~ms}, S E=17.49)$ trials, as well as the main effect of Distractor type, $F(1,19)=5.642, p=.028, \eta_{p}^{2}=$ .229 , since the facial stimulus elicited smaller latencies $(M=$ $239 \mathrm{~ms}, S E=16.82)$ as compared to the arrow stimulus $(M=$ $256 \mathrm{~ms}, S E=16.16)$. Importantly, the interaction was nonsignificant, $F(1,19)<1, p=.996, \eta_{p}^{2}<.001$, indicating that the magnitude of the interference effect elicited by the two distractors was similar (see also Fig. 2). Bayesian analyses indicated that the model including only Congruency and Distractor type as main effects was the best model fitting the

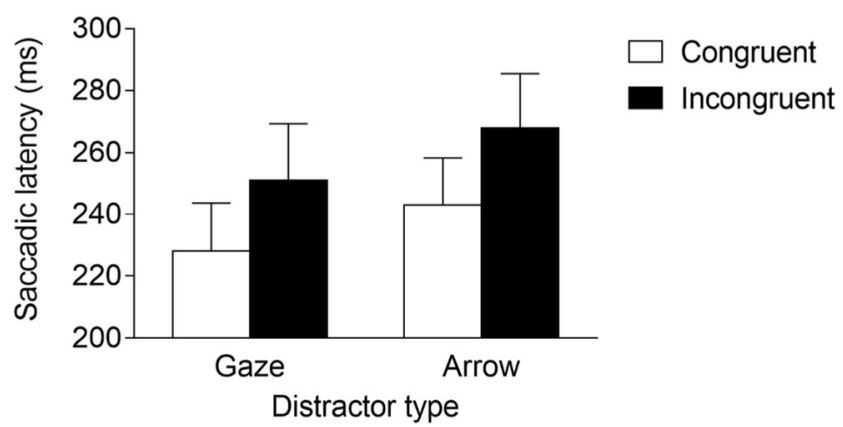

Fig. 2 Mean saccadic latencies as a function of the different experimental conditions in Experiment 1. Error bars are SEM data and was also preferable to the model including the interaction term, $B F_{10}=3.38$.

With regard to errors, the main effects of Congruency, $F(1$, 19) $=1.876, p=.187, \eta_{p}^{2}=.09$, Distractor type, $F(1,19)=$ $1.804, p=.195, \eta_{p}^{2}=.087$, and their interaction, $F(1,19)<1$, $p=.848, \eta_{p}^{2}=.002$, were all non-significant (see also Table 1). Bayesian analyses confirmed that the null model was the best model fitting the data and was also preferable to the model including the interaction term, $B F_{10}=16.13$.

\section{Discussion}

The main result of this experiment was a clear interference effect reflected in saccadic latencies. This means that, even if saccades were likely to be prepared well in advance, within a temporal window that abundantly preceded the onset of the distractor stimulus, the distractor was still able to exert an influence on the oculomotor system. Importantly, the interference effect was similar for both gaze and arrow distractors, thus aligning with previous studies reporting comparable results for these two stimuli (e.g., Kuhn \& Kingstone, 2009). Nevertheless, we reasoned that, since the direction word was provided on a trial-by-trial basis, participants were required to constantly update the spatial location towards which they had to execute the instructed saccade. Hence, saccades were prepared within a variable context that may have allowed the distractor to penetrate the participant's attentional set and therefore influence her/his eye movement behavior. A second experiment was therefore carried out in which the direction word was kept constant within a block of trials. We hypothesized that the adoption of this fixed and unequivocal preparatory context could provide an even stronger test of the power of distractor stimuli to exert their interference on saccade execution, in that the participants were allowed to maintain a structured and rigid attentional set that should further shield them against intrusions from task-irrelevant distractor stimuli.

\section{Experiment 2: Blocked execution of leftward and rightward saccades}

\section{Methods \\ Participants}

A new sample of 20 students naïve to the study (mean age $=$ 24 years, $S D=1.53$, seven males) was tested. They had normal or corrected-to-normal vision and provided written, informed consent. No compensation or credits were given. The study was approved by the Ethics Committee for Psychological Research at the University of Padova and conducted in accordance with the Declaration of Helsinki. 
Table 1 Mean saccadic latencies (in ms) and percentage of errors observed in all the experimental conditions of Experiments 1 and 2. Values in parentheses are SEM

\begin{tabular}{|c|c|c|c|c|}
\hline & \multicolumn{2}{|l|}{ Gaze } & \multicolumn{2}{|l|}{ Arrow } \\
\hline & Congruent & Incongruent & Congruent & Incongruent \\
\hline \multicolumn{5}{|l|}{ Experiment 1} \\
\hline Saccadic latencies & $228(15.62)$ & $251(18.35)$ & $243(15.26)$ & $268(17.47)$ \\
\hline Saccadic errors & $2.657(.728)$ & $3.453(.836)$ & $2.061(.792)$ & $2.602(.585)$ \\
\hline \multicolumn{5}{|l|}{ Experiment 2} \\
\hline Saccadic latencies & $225(14.65)$ & $241(14.03)$ & $250(13.92)$ & $275(13.15)$ \\
\hline Saccadic errors & $2.412(.946)$ & $3.136(1.403)$ & $1.122(.491)$ & $2.456(.887)$ \\
\hline
\end{tabular}

\section{Apparatus and stimuli}

Both apparatus and stimuli were the same as those employed in Experiment 1.

\section{Procedure}

The procedure was identical to that employed in Experiment 1, with the following exception: saccade instruction was delivered in a blocked fashion. More specifically, half of the participants completed four different blocks of trials (two blocks for each distractor stimulus) in which saccadic direction was kept constant (i.e., Block 1: LEFT; Block 2: RIGHT; Block 3: LEFT; Block 4: RIGHT). The remaining participants completed the four blocks in the opposite order (i.e., Block 1: RIGHT; Block 2: LEFT; Block 3: RIGHT; Block 4: LEFT). As in Experiment 1, eye-gaze and arrow distractors were presented in two distinct blocks and block order was counterbalanced across participants.

\section{Data handling and analyses}

Data were handled and analyzed as in Experiment 1. Mean saccadic amplitude was $9.79^{\circ}$. Saccadic errors were low $(2.3 \%)$ and analyzed separately.

\section{Results}

With regard to latencies, the main effect of Congruency was significant, $F(1,19)=13.540, p=.002, \eta_{p}^{2}=.416$, with smaller latencies on congruent $(M=238 \mathrm{~ms}, S E=13.46)$ than on incongruent $(M=258 \mathrm{~ms}, S E=12.74)$ trials, as well as the main effect of Distractor type, $F(1,19)=13.162, p=.002, \eta_{p}^{2}$ $=.409$, since the facial stimulus elicited smaller latencies $(M=$ $233 \mathrm{~ms}, S E=14.12)$ as compared to the arrow stimulus $(M=$ $263 \mathrm{~ms}, S E=13.21)$. Importantly, the interaction was nonsignificant, $F(1,19)<1, p=.452, \eta_{p}^{2}=.030$, indicating that the magnitude of the interference effect elicited by the two distractors was similar (see also Fig. 3). Bayesian analyses indicated that the model including only Congruency and Distractor type as main effects was the best model fitting the data and was also preferable to the model including the interaction term, $B F_{10}=3.1$.

With regard to errors, the main effects of Congruency, $F(1$, 19) $=1.837, p=.191, \eta_{p}^{2}=.088$, Distractor type,$F(1,19)=$ 2.443, $p=.135, \eta_{p}^{2}=.114$, and their interaction, $F(1,19)<1$, $p=.503, \eta_{p}^{2}=.024$, were all non-significant (see also Table 1). Bayesian analyses confirmed that the null model was the best model fitting the data and was also preferable to the model including the interaction term, $B F_{10}=6.06$.

\section{Discussion}

The main result obtained in this second experiment was virtually identical to that of Experiment 1. Indeed, the interference effect that emerged in saccadic latencies was similar in magnitude for both gaze and arrow distractors. Hence, not even the adoption of an unequivocal and blocked preparatory context for saccadic eye movements was able to override the influence of the distracting stimuli.

\section{General discussion}

In two experiments, we assessed whether the reflexive tendency to perform saccades following the direction of others' eye-

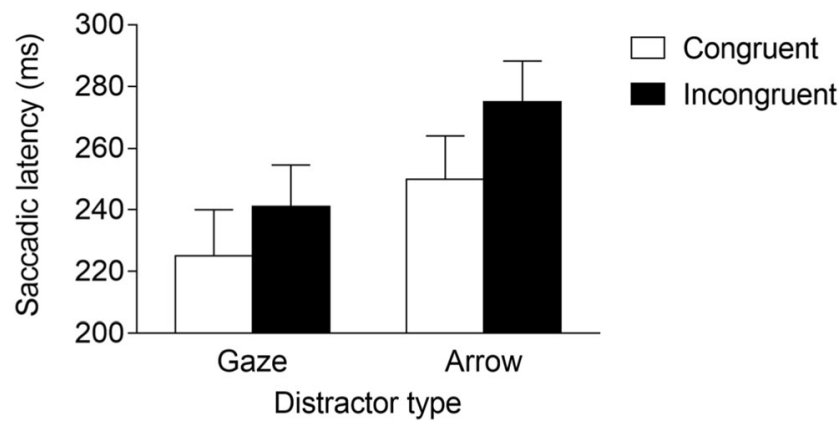

Fig. 3 Mean saccadic latencies as a function of the different experimental conditions in Experiment 2. Error bars are SEM 
gaze direction can be abolished when participants are allowed to prepare saccades before the task-irrelevant eye-gaze stimulus is encountered. More specifically, the instruction to perform a leftward or a rightward saccade was delivered at the beginning of each trial in either an intermixed (Experiment 1) or a blocked (Experiment 2) fashion, and only after more than 2,000 $\mathrm{ms}$ did the gaze stimulus distractor appear alongside the go signal to execute the requested saccade. This means that participants had a reasonable time for programming the saccadic eye movements before the onset of the distractor. Arrows were also employed as non-social control stimuli. In both experiments, the results showed that, even if saccades were likely to be prepared within a temporal window that preceded the onset of the distracting stimulus, distractors were still able to interfere with the execution of the planned eye movement. Indeed, saccadic latencies were smaller on spatially congruent than on spatially incongruent trials. Moreover, the interference effect was similar for both gaze and arrow distractors, in line with previous studies reporting comparable attentional effects elicited by these two types of stimuli on both oculomotor and manual responses (e.g., Kuhn \& Kingstone, 2009; Tipples, 2008). Additionally, the interference effect was not reflected in saccadic errors - these were particularly rare (i.e., about $2 \%$ of the trials in both experiments) - indicating that participants were overall proficient in launching the saccade towards the programmed direction. It might be argued that participants could have potentially waited for the go signal before preparing the saccadic eye movement, although this would have been dysfunctional in relation to an optimal performance in the task. While we have no direct evidence to definitely rule out this possibility, we tend to discard it based on the fact that, if this were the case, then (1) one might have expected higher overall saccadic latencies (regardless of congruency) as compared to those reported in the present study, and (2) a significant oculomotor interference effect should also have emerged in saccadic errors, as reported in previous studies using this paradigm (see, e.g., Kuhn et al., 2010; Kuhn \& Kingstone, 2009).

The current experiments do not allow us to address the specific effects of preparation within the task and point to an opportunity for future research in which a baseline without prior knowledge about the direction of the instructed saccade is included. This will enable estimation of whether and how the lack of preparation can lead to magnified oculomotor interference effects. Future studies might also include a range of time intervals separating the averted gaze/arrow stimuli and the go signal in order to shed light on the time needed to overcome the automatic effects of task-irrelevant signals (see, e.g., Theeuwes, Atcheley, \& Kramer, 2000).

Another result that emerged in both experiments was that latencies were overall smaller in the presence of the eye-gaze than the arrow distractor, in line with some previous studies (see Kuhn et al., 2010). Although unexpected, this pattern seems to align with a recent work reporting that eye contact episodes with a real task-irrelevant individual produced faster responses to peripheral targets as compared to a condition in which eye contact was absent (Hietanen, Myllyneva, Helminen, \& Lyyra, 2016). According to Hietanen and colleagues, this would have likely been caused by the increased levels of arousal that can be evidenced when looking another person directly in the eyes (see also Hietanen, 2018). An alternative account would instead ascribe the difference in the observed pattern to the qualitatively different way we might process gaze and arrow stimuli (e.g., Marotta, Lupiáñez, Martella, \& Casagrande, 2012). However, despite this effect, the oculomotor interference generated by these two stimuli had a similar magnitude, thus suggesting that both social and non-social distractors can exert a strong influence on saccade execution.

The present study is also embedded in a recent stream of oculomotor research in which relevant factors are manipulated within the same block of trials on the one hand, or in different blocks on the other hand (e.g., Dalmaso, Castelli, \& Galfano, 2019; Dalmaso, Castelli, \& Galfano, 2020c; Zeligman \& Zivotofsky, 2017). One specific manner in which this goal can be achieved is by manipulating experimental instructions in such a way that the participants are forced to adopt either a broad or a narrow attentional set depending on whether their knowledge about a taskrelevant feature (e.g., the direction of the instructed saccade or the target location) is kept constant within a block of trials or changes unpredictably from trial to trial. For instance, Zeligman and Zivotofsky (2017) found that participants had a better performance in an oculomotor task when different directional instructions were provided in a blocked rather than an intermixed fashion. Overall, it seems that blocked designs can help in establishing a particularly adequate context within which participants are much more proficient in focusing on the instruction related to the oculomotor task at hand. This, in turn, could help participants to shield against task-irrelevant information arising from the experimental setting. In this perspective, the results of the present experiments suggest that the spatial direction conveyed by both gaze and arrow is something that individuals can hardly ignore, and speaks in favor of a rather strong automatic processing of these two kinds of spatially connoted stimuli that elicited oculomotor interference even when participants not only had plenty of time to program the correct saccade in advance but were also aware that the spatial vector of the instructed saccade remained constant throughout an entire block of trials. These arguments do not imply that both gaze and arrow distractors always exert an impact to the same extent, independent of other situational aspects. For instance, Marotta et al. (2012) have shown that objectbased attentional orienting occurs for arrow but not for 
gaze cues. Moreover, other evidence comes from studies in which arrows and gaze were used as targets, instead of as distractor stimuli (e.g., Marotta, Román-Caballero, \& Lupiáñez, 2018). Importantly, in the case of gaze stimuli, relevant high-level social factors (e.g., the group membership of the faces providing an averted gaze) can additionally modulate attentional processes. This is consistent with a view that conceptualizes attentional effects in terms of conditionally automatic processes that inevitably occur when specific preconditions are met (Dalmaso et al., 2020b). However, when no other factors, as in the present study, prompt selectively prioritizing some stimuli over others as a function of the social meaning they convey, following the gaze of other individuals appears to be the default response.

To sum up, the present results provide further evidence that the influence of eye-gaze and arrow distractors on saccadic eye movements cannot entirely be suppressed, even when the processes underlying saccade preparation can operate well in advance with respect to the temporal window in which participants are presented with both the go signal to execute the saccade and the distractor. This, in turn, suggests that the information provided by this type of stimuli is highly prioritized by the attention system, consistent with the notion that gaze and arrows are among the most powerful signals for eliciting spatial orienting.

Acknowledgements We are grateful to Francesco Naccari for his assistance in data collection, and to James Brockmole, Deborah Cronin, and Wieske Van Zoest for their valuable comments on a previous version of this article. This study was supported by a DPSS-SID2019 grant (University of Padova) to MD.

Open Practices Statement The data and stimuli are available on OSF at https://doi.org/10.17605/OSF.IO/Q6CBK and the study was not preregistered.

\section{References}

Capozzi, F., \& Ristic, J. (2018). How attention gates social interactions. Annals of the New York Academy of Sciences, 1426, 179-198. https://doi.org/10.1111/nyas.13854

Ciardo, F., Marino, B. F. M., Actis-Grosso, R., Rossetti, A., \& Ricciardelli, P. (2014). Face age modulates gaze following in young adults. Scientific Reports, 4, 4746. https://doi.org/10.1038/ srep04746

Dalmaso, M., Alessi, G., Castelli, L., \& Galfano, G. (2020a). Eye contact boosts the reflexive component of overt gaze following. Scientific Reports, 10, 4777. https://doi.org/10.1038/s41598-020-61619-6

Dalmaso, M., Castelli, L., \& Galfano, G. (2019). Self-related shapes can hold the eyes. Quarterly Journal of Experimental Psychology, 72, 2249-2260. https://doi.org/10.1177/1747021819839668

Dalmaso, M., Castelli, L., \& Galfano, G. (2020b). Social modulators of gaze-mediated orienting of attention: A review. Psychonomic Bulletin \& Review. https://doi.org/10.3758/s13423-020-01730-x

Dalmaso, M., Castelli, L., \& Galfano, G. (2020c). Microsaccadic rate and pupil size dynamics in pro-/anti-saccade preparation: the impact of intermixed vs. blocked trial administration. Psychological Research, 84, 1320-1332. https://doi.org/10.1007/s00426-018-01141-7

Dalmaso, M., Galfano, G., \& Castelli, L. (2015). The impact of same- and other-race gaze distractors on the control of saccadic eye movements. Perception, 44, 1020-1028. https://doi.org/10.1177/ 0301006615594936

Galfano, G., Dalmaso, M., Marzoli, D., Pavan, G., Coricelli, C., \& Castelli, L. (2012). Eye gaze cannot be ignored (but neither can arrows). Quarterly Journal of Experimental Psychology, 65, 18951910. https://doi.org/10.1080/17470218.2012.663765

Guzzon, D., Brignani, D., Miniussi, C., \& Marzi, C. A. (2010). Orienting of attention with eye and arrow cues and the effect of overtraining. Acta Psychologica, 134, 353-362. https://doi.org/10.1016/j.actpsy. 2010.03.008

Hermens, F., \& Walker, R. (2010). Gaze and arrow distractors influence saccade trajectories similarly. Quarterly Journal of Experimental Psychology, 63, 2120-2140. https://doi.org/10.1080/ 17470211003718721

Hietanen, J. K. (2018). Affective eye contact: an integrative review. Frontiers in Psychology, 9, 1587. https://doi.org/10.3389/fpsyg. 2018.01587

Hietanen, J. K., Myllyneva, A., Helminen, T. M., \& Lyyra, P. (2016). The effects of genuine eye contact on visuospatial and selective attention. Journal of Experimental Psychology: General, 145, 11021106. https://doi.org/10.1037/xge0000199

Hommel, B., Pratt, J., Colzato, L., \& Godijn, R. (2001). Symbolic control of visual attention. Psychological Science, 12, 360-365. https://doi. org/10.1111/1467-9280.00367

Howell, D. C. (2013). Statistical methods for psychology. Belmont, CA: Wadsworth Cengage Learning.

JASP Team (2020). JASP (Version 0.12.2) [Computer software].

Kuhn, G., \& Benson, V. (2007). The influence of eye-gaze and arrow pointing distractor cues on voluntary eye movements. Perception \& Psychophysics, 69, 966-971. Retrieved from https://www.ncbi.nlm. nih.gov/pubmed/18018978

Kuhn, G., Benson, V., Fletcher-Watson, S., Kovshoff, H., McCormick, C. A., Kirkby, J., \& Leekam, S. R. (2010). Eye movements affirm: Automatic overt gaze and arrow cueing for typical adults and adults with autism spectrum disorder. Experimental Brain Research, 201, 155-165. https://doi.org/10.1007/s00221-009-2019-7

Kuhn, G., \& Kingstone, A. (2009). Look away! Eyes and arrows engage oculomotor responses automatically. Attention, Perception, and Psychophysics, 71, 314-327. https://doi.org/10.3758/APP.71.2.314

Marotta, A., Lupiáñez, J., Martella, D., \& Casagrande, M. (2012). Eye gaze versus arrows as spatial cues: Two qualitatively different modes of attentional selection. Journal of Experimental Psychology: Human Perception and Performance, 38, 326-335. https://doi.org/10.1037/a0023959

Marotta, A., Román-Caballero, R., \& Lupiáñez, J. (2018). Arrows don’t look at you: Qualitatively different attentional mechanisms triggered by gaze and arrows. Psychonomic Bulletin \& Review, 25, 22542259. https://doi.org/10.3758/s13423-018-1457-2

Porciello, G., Liuzza, M. T., Minio-Paluello, I., Caprara, G. V., \& Aglioti, S. M. (2016). Fortunes and misfortunes of political leaders reflected in the eyes of their electors. Experimental Brain Research, 234, 733740. https://doi.org/10.1007/s00221-015-4496-1

Ricciardelli, P., Bricolo, E., Aglioti, S., \& Chelazzi, L. (2002). My eyes want to look where your eyes are looking: Exploring the tendency to imitate another individual's gaze. Neuroreport, 13, 2259-2264. https://doi.org/10.1097/01.wnr.0000044227

Tatler, B. W., Wade, N. J., Kwan, H., Findlay, J. M., \& Velichkovsky, B. M. (2010). Yarbus, eye movements, and vision. I-Perception, 1, 727. https://doi.org/10.1068/i0382

Theeuwes, J., Atchley, P., \& Kramer, A. F. (2000). On the time course of top-down and bottom-up control of visual attention. In J. Driver 
(Ed.), Attention \& Performance, vol. 18. (pp. 105-125). Cambridge: MIT Press.

Tipples, J. (2008). Orienting to counterpredictive gaze and arrow cues. Perception \& Psychophysics, 70, 77-87. https://doi.org/10.3758/PP. 70.1 .77

Yarbus, A. L. (1967). Eye Movements and Vision. Boston, MA: Springer US. https://doi.org/10.1007/978-1-4899-5379-7
Zeligman, L., \& Zivotofsky, A. Z. (2017). Back to basics: The effects of block vs. interleaved trial administration on pro-and anti-saccade performance. PLoS ONE, 12, e0172485. https://doi.org/10.1371/ journal.pone. 0172485

Publisher's note Springer Nature remains neutral with regard to jurisdictional claims in published maps and institutional affiliations. 